\title{
Real time electrocardiogram QRS detection using combined adaptive threshold
} Ivaylo I Christov*

\author{
Address: Center of Biomedical Engineering, Bulgarian Academy of Sciences, Acad. G. Bonchev str., blok 105, 1113, Sofia, Bulgaria \\ Email: Ivaylo I Christov* - Ivaylo.Christov@clbme.bas.bg \\ * Corresponding author
}

Published: 27 August 2004

BioMedical Engineering OnLine 2004, 3:28 doi:10.1186/1475-925X-3-28
Received: 04 June 2004

Accepted: 27 August 2004

This article is available from: http://www.biomedical-engineering-online.com/content/3/I/28

(c) 2004 Christov; licensee BioMed Central Ltd.

This is an open-access article distributed under the terms of the Creative Commons Attribution License (http://creativecommons.org/licenses/by/2.0), which permits unrestricted use, distribution, and reproduction in any medium, provided the original work is properly cited.

\begin{abstract}
Background: QRS and ventricular beat detection is a basic procedure for electrocardiogram (ECG) processing and analysis. Large variety of methods have been proposed and used, featuring high percentages of correct detection. Nevertheless, the problem remains open especially with respect to higher detection accuracy in noisy ECGs
\end{abstract}

Methods: A real-time detection method is proposed, based on comparison between absolute values of summed differentiated electrocardiograms of one of more ECG leads and adaptive threshold. The threshold combines three parameters: an adaptive slew-rate value, a second value which rises when high-frequency noise occurs, and a third one intended to avoid missing of low amplitude beats.

Two algorithms were developed: Algorithm I detects at the current beat and Algorithm 2 has an $R R$ interval analysis component in addition.

The algorithms are self-adjusting to the thresholds and weighting constants, regardless of resolution and sampling frequency used. They operate with any number $L$ of ECG leads, selfsynchronize to QRS or beat slopes and adapt to beat-to-beat intervals.

Results: The algorithms were tested by an independent expert, thus excluding possible author's influence, using all 48 full-length ECG records of the MIT-BIH arrhythmia database. The results were: sensitivity $\mathrm{Se}=99.69 \%$ and specificity $\mathrm{Sp}=99.65 \%$ for Algorithm I and $\mathrm{Se}=99.74 \%$ and $S p=99.65 \%$ for Algorithm 2 .

Conclusion: The statistical indices are higher than, or comparable to those, cited in the scientific literature.

\section{Background}

The QRS complexes and ventricular beats in an electrocardiogram represent the depolarization phenomenon of the ventricles and yield useful information about their behavior. Beat detection is a procedure preceding any kind of ECG processing and analysis. For morphological analysis this is the reference for detection of other ECG waves and parameter measurements. Rhythm analysis requires classification of QRS and other ventricular beat complexes as normal and abnormal. Real-time ventricular beat detection is essential for monitoring of patients in critical heart condition. 
Correct beats recognition is impeded by power-line interference, electromyogram noise and baseline wander often present in the ECG signal.

In long-term monitoring electrode impedance can increase considerably, resulting in very low signal-tonoise ratio, which can make detection practically impossible in a single lead. Therefore, usually two or three leads are used for monitoring [1].

Friesen et al. [2] have presented a comparison of nine QRS detection algorithms, based on: i) amplitude and first derivative, ii) first derivative only, iii) first and second derivative, and iv) digital filtering. Daskalov et al. [3] applied these algorithms to selected signals containing records with pronounced baseline drift. The results were unsatisfactory, which was probably due to the use of fixed detection thresholds, whereas adaptive ones would be more appropriate.

Poli et al. [4] used a generic algorithm for QRS detection. The complexes were emphasized with respect to the rest of the signal by polynomial filters and compared to an adaptive threshold. The authors reported $99.60 \%$ sensitivity (Se) and $99.51 \%$ specificity (Sp) with the MIT-BIH Arrhythmia Database. The method is inapplicable in realtime.

Afonso et al. [5] proposed hardware filter banks for ECG signal decomposition, where several parameters were independently computed and combined in a decision rule. The authors reported $\mathrm{Se}=99.59 \%$ and $\mathrm{Sp}=99.56 \%$ for their real-time, single-channel beat detection algorithm tested with the MIT-BIH Arrhythmia Database.

Dotsinsky and Stoyanov [6] developed a heuristic, pseudo-real-time algorithm for ventricular beat detection for single-channel ECG, based on steep edges and sharp peaks evaluation criteria. They reported $\mathrm{Se}=99.04 \%$ and $\mathrm{Sp}=99.62 \%$, obtained with two channel recordings from AHA and MIT-BIH Arrhythmia Database

Moraes et al. [1] combined logically two different algorithms working in parallel - the first has been taken from the work of Englese and Zeelenberg [7] and the other was based on Pan and Tompkins [8], and Ligtenberg and Kunt [9]. Moraes et al. [1] reported $\mathrm{Se}=99.22 \%$ and $\mathrm{Sp}=99.73$ $\%$ after having excluded records of patients with pacemaker. After excluding a few more recordings 108, 200, 201 and 203, containing high amplitude noise (according to the authors), the statistical indices rises to $\mathrm{Se}=99.56 \%$ and $\mathrm{Sp}=99.82 \%$.

Li et al. [10] have used wavelet transforms for detection. They reported $0.15 \%$ false detections out of 46 files from the MIT-BIH Arrhythmia Database, but with exclusion of files 214 and 215. In addition, we found some errors in their Table II. After correction, the reported accuracy slightly decreased.

The large variety of QRS detection algorithms, and the continuous efforts for their enhancement, proves that universally acceptable solution has not been found yet. Difficulties arise mainly from the huge diversity of the QRS complex waveforms and the noise and artifacts accompanying the ECG signals.

\section{ECG databases}

All 48 ECG recordings of MIT-BIH Arrhythmia database were used, without exception. Each one has a duration of $30 \mathrm{~min}$ and includes two leads - the modified limb lead II and one of the modified leads V1, V2, V4 or V5 [11]. The sampling frequency is $360 \mathrm{~Hz}$ with resolution $5 \mu \mathrm{V} /$ bit. Two cardiologists have annotated all beats. Approximately $70 \%$ of the beats are annotated as Normal. Four of the records are from patients with pacemakers.

The American Heart Association (AHA) database was also considered, during the evaluation of the method, mostly due to the fact that it contains patients with premature ventricular beats of contraction of R-over-T type, some of them very difficult to detect because of their closeness to the previous complex. Statistical indices for this database are not derived, because they can be compared with limited number of articles working with AHA. The database consists of 80 recordings: 2-leads, $250 \mathrm{~Hz}$ sampling rate and $5 \mu \mathrm{V} / \mathrm{bit}$ resolution.

\section{Method}

The differentiated and summed signals from $L$ leads are compared to the absolute value of a threshold $M F R=M+$ $F+R-$ a combination of three independent adaptive thresholds, where:

- $M$ - Steep-slope threshold;

- F - Integrating threshold for high-frequency signal components;

- $R$ - Beat expectation threshold.

Two algorithms were developed:

Algorithm 1 detects at the current beat.

Algorithm 2 Pseudo-real-time detection with additional triggering of potentially missed heart beat in the last interval by RR interval analyses. 
The algorithms are self-adjusting to the thresholds and weighting constants, regardless of resolution and sampling frequency used. They operate with any number $L$ of ECG leads, self-synchronize to QRS or beat slopes and adapt to beat-to-beat intervals.

\section{Preprocessing}

- Moving averaging filter for power-line interference suppression: averages samples in one period of the powerline interference frequency with a first zero at this frequency.

- Moving averaging of samples in $28 \mathrm{~ms}$ interval for electromyogram noise suppression - a filter with first zero at about $35 \mathrm{~Hz}$.

- Moving averaging of a complex lead (the sintesis is explained in the next section) in $40 \mathrm{~ms}$ intervals - a filter with first zero at about $25 \mathrm{~Hz}$. It is suppressing the noise magnified by the differentiation procedure used in the process of the complex lead sintesis.

\section{Complex lead}

The algorithm operates with a complex lead $Y$ of several primary leads $L$. In cases of 12-standard leads, synthesis of the three quasi-orthogonal Frank leads is recommended first $[3,12]$, thus determining the complex lead as a spatial vector. The complex lead is obtained as:

$\mathrm{Y}(\mathrm{i})=\frac{1}{L} \sum_{\mathrm{j}=1}^{\mathrm{L}} \mathrm{abs}(\mathrm{Xj}(\mathrm{i}+1)-\mathrm{Xj}(\mathrm{i}-1))$

where $X j(i)$ is the amplitude value of the sample $i$ in lead $j$, and $Y(i)$ is the current complex lead.

The above formula (except the normalizing coefficient 1/ $\mathrm{L}$ and the absolute value) was initially adopted from the work of Bakardjian [13]. Operating with unsigned (absolute) values proved convenient when dealing with QRSs and extrasystoles having different, for example positive (in one lead) and negative (in the other lead) deflections.

\section{Adaptive steep-slope threshold - M}

- Initially $M=0.6^{*} \max (Y)$ is set for the first $5 \mathrm{~s}$ of the signal, where at least 2 QRS complexes should occur. A buffer with 5 steep-slope threshold values is preset:

$M M=\left[M_{1} M_{2} M_{3} M_{4} M_{5}\right]$,

where $M_{1} \div M_{5}$ are equal to $M$.

- QRS or beat complex is detected if $Y i \geq M F R$,
- No detection is allowed $200 \mathrm{~ms}$ after the current one. In the interval $\mathrm{QRS} \div \mathrm{QRS}_{+200 m s}$ a new value of $M_{5}$ is calculated:

$n e w M_{5}=0.6 * \max (Y i)$

The estimated new $M_{5}$ value can become quite high, if steep slope premature ventricular contraction or artifact appeared, and for that reason it is limited to new $M_{5}=1.1^{*}$ $M_{5}$ if new $M_{5}>1.5^{*} M_{5}$.

The MM buffer is refreshed excluding the oldest component, and including $M_{5}=n e w M_{5} . M$ is calculated as an average value of $M M$.

- $M$ is decreased in an interval 200 to $1200 \mathrm{~ms}$ following the last QRS detection at a low slope, reaching $60 \%$ of its refreshed value at $1200 \mathrm{~ms}$.

- After 1200 ms M remains unchanged.

The thresholds definitions are presented in more detail with the help of several examples. Two ECG leads are shown in Fig. 1a. Detected QRSs are marked with 'red O' on Lead 1. The summary lead and the steep-slope threshold are represented in Fig. 1b.

\section{Adaptive integrating threshold - F}

The integrating threshold $F$ is intended to raise the combined threshold if electromyogram noise is accompanying the ECG, thus protecting the algorithm against 'erroneous beat detection'.

Initially $F$ is the mean value of the pseudo-spatial velocity $Y$ for $350 \mathrm{~ms}$.

With every signal sample, $F$ is updated adding the maximum of $Y$ in the latest $50 \mathrm{~ms}$ of the $350 \mathrm{~ms}$ interval and subtracting $\max Y$ in the earliest $50 \mathrm{~ms}$ of the interval.

$\mathrm{F}=\mathrm{F}+\left(\max \left(\mathrm{Y}_{\text {in latest }} 50 \mathrm{~ms}\right.\right.$ in the $350 \mathrm{~ms}$ interval $)-\max \left(\mathrm{Y}_{\text {in earliest }}\right.$ $50 \mathrm{~ms}$ in the $350 \mathrm{~ms}$ interval $)$ )/150

The way $F$ is updated means that not every sample in the interval is integrated, but just the envelope of the pseudospatial velocity $Y$. The weight coefficient $1 / 150$ is empirically derived.

Two ECG leads are shown in Fig. 2a. The pseudo-spatial velocity $Y$ and the integrated threshold are presented in Fig. $2 b$. The correct detection is due to the rise of $F$ (hence of MFR) with about $0.2 \mathrm{mV}$. The beat complex is included in the integration process (note the high rise of $F$ after any of the complexes), thus making almost impossible a close detection to the previous complex. 

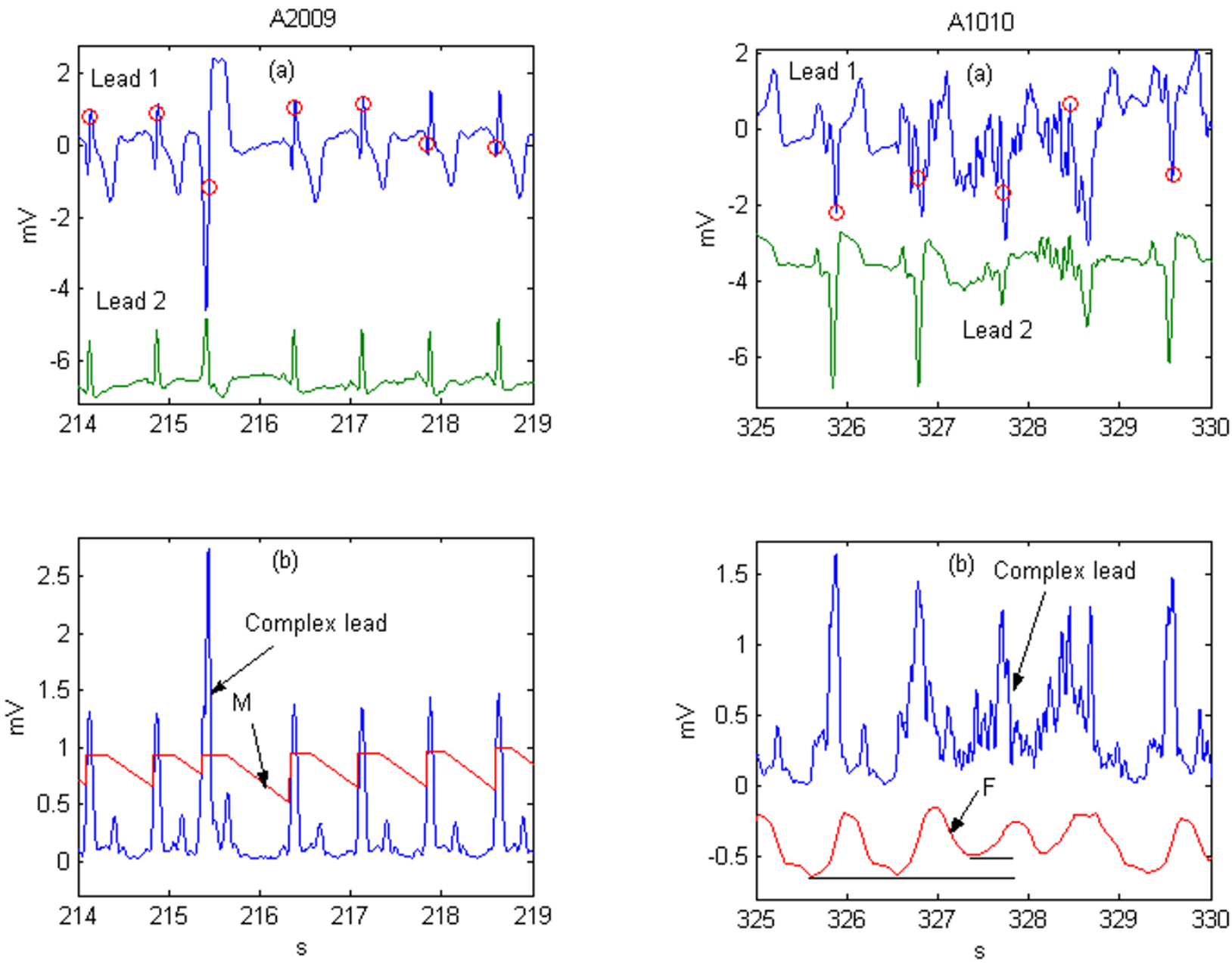

Figure I

Adaptive steep-slope threshold

\section{Adaptive beat expectation threshold - $\mathbf{R}$}

The beat expectation threshold $R$ is intended to deal with heartbeats of normal amplitude followed by a beat with very small amplitude (and respectively with very small slew rate). This can be observed for example in cases of electrode artifacts. Conversely to the integrating threshold protecting against erroneous QRS detection, $R$ is protecting against 'QRS misdetection'.

A buffer with the 5 last RR intervals is updated at any new QRS detection. $R m$ is the mean value of the buffer.

- $R=0 \mathrm{~V}$ in the interval from the last detected QRS to $2 / 3$ of the expected $R m$.

- In the interval QRS + Rm * 2/3 to QRS + Rm, $R$ decreases 1.4 times slower then the decrease of the previously discussed steep slope threshold ( $M$ in the 200-1200 ms interval).

- After QRS + Rm the decrease of $R$ is stopped.

The time-course of the beat expectation threshold $R$ is shown in Fig. 3. The decrease of $R$ (respectively MFR) with about $0.2 \mathrm{mV}$ at the fourth QRS allows its detection, despite the lack of complex in Lead 2, which leads to a two-fold decrease of the summary lead amplitude $Y$ (Fig. $3 b)$. 

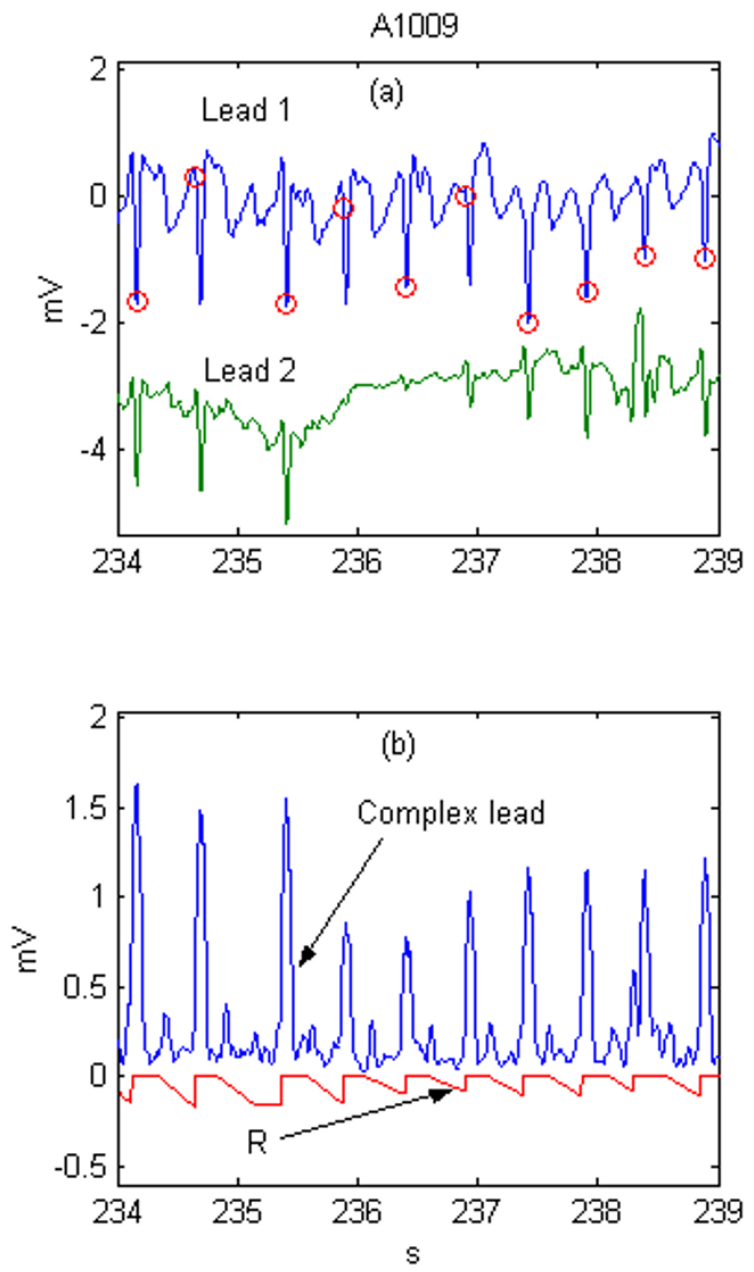

Figure 3

Adaptive beat expectation threshold

\section{Combined adaptive threshold - MFR}

The combined adaptive threshold is a sum of the adaptive steep-slope threshold, adaptive integrating threshold and adaptive beat expectation thresholds. (Fig. 4)

$M F R=M+F+R$

\section{Algorithm 2: pseudo-real-time detection with additional triggering of eventually missed heart beat in the last detected RR interval}

All previous considerations relate to Algorithm 1, which detects a beat at its occurrence. Additional checking for an eventually missed heartbeat is performed by Algorithm 2 . Its function is explained by the signal in Fig. 5. The fourth
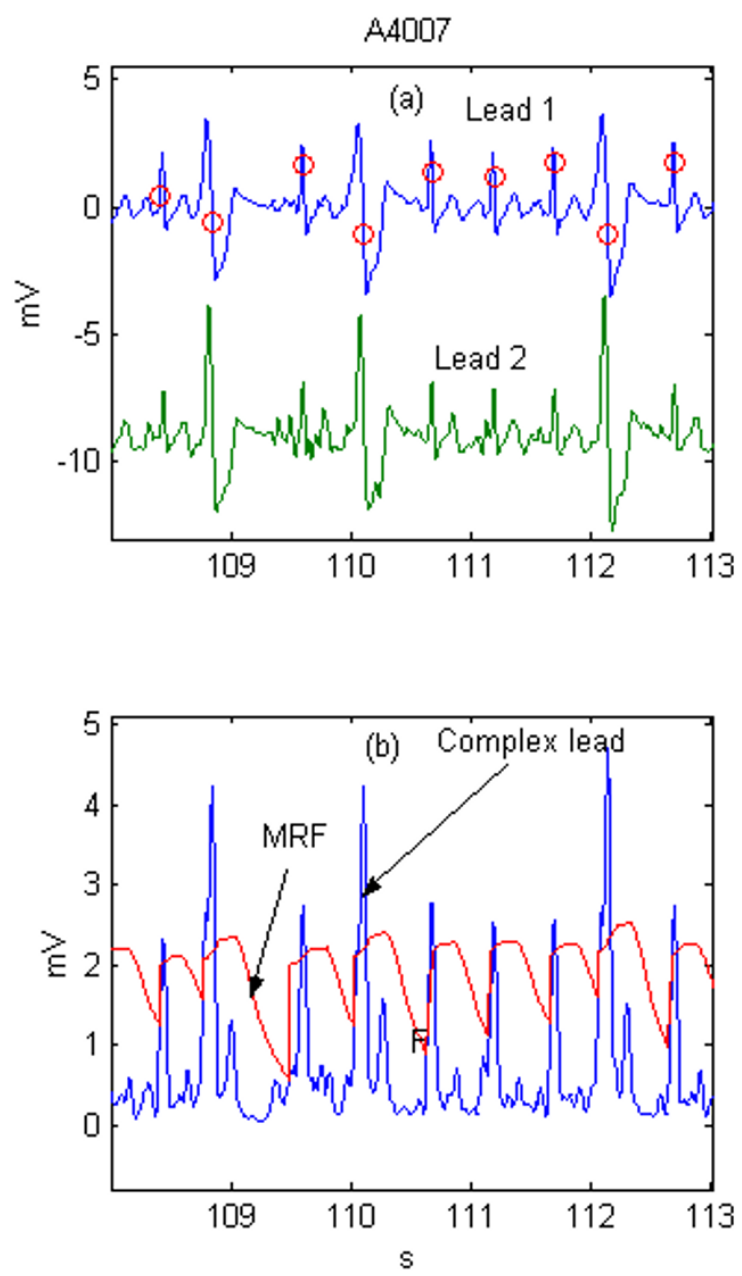

Figure 4

Combined adaptive threshold

complex at the $15.2 \mathrm{~s}$ in Fig. $5 \mathrm{~b}$ should be missed due to the fact that, MFR is greater then the summary lead $Y$.

Let's mark the previous RR interval with $t 1$ and the last with $t 2$ (Fig. 5a).

If $t 1$ is not shortened, which is tested by logic OR of the 2 conditions $\mathrm{t} 1>\mathrm{Rm}$ OR Rm- $\mathrm{t} 1<0.12 * \mathrm{Rm}$ AND in the same time $t 2$ is quite long to fulfill the condition abs(t2$2 * \mathrm{Rm})<0.5 * \mathrm{Rm}$, the interval is subjected to check for a missed complex.

A test is performed on each of the primary leads where a sharp peak is searched (defined as a product $>4 \mu \mathrm{V}$ of two 

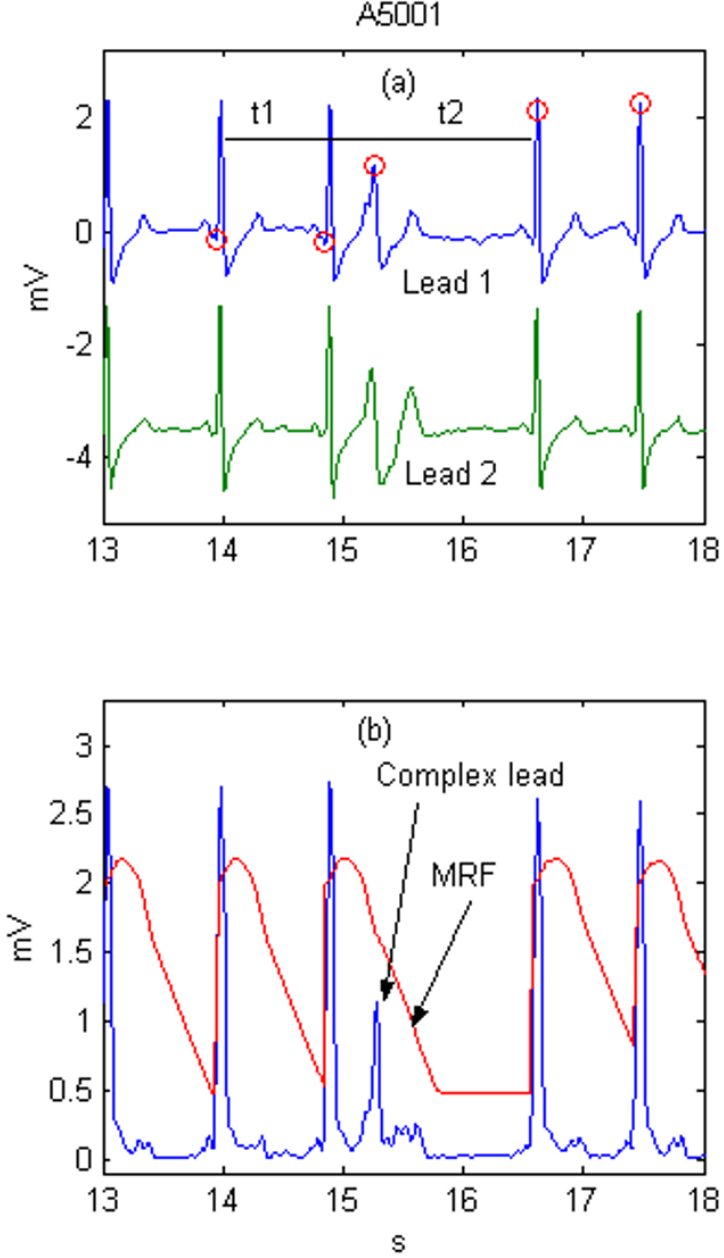

Figure 5

Pseudo-real-time detection with additional triggering of eventually missed heart beat in the last RR interval.

signal differences having one central and two lateral points $8 \mathrm{~ms}$ apart). If the test is passed, a second one is carried out for the amplitude of the summary lead at that point, which should be bigger then $1 / 3$ of the mean value of the buffer $M M$, in order to define this point as a missed QRS complex.

\section{Results and discussion}

Normally the statistical indices Se and Sp are derived from the following parameters: correctly detected beats TP (true positive), falsely detected beats FP (false positive) and undetected beats FN (false negative). In addition, we used two parameters, adopted from Dotsinsky and Stoyanov [6], as described below.

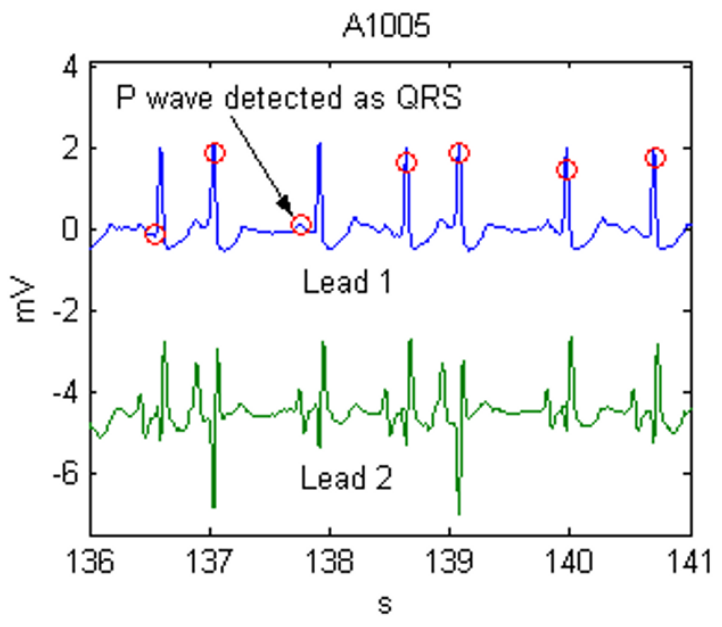

Figure 6

Shifted positive error at the $P$ wave

SP - shifted positive error was introduced in order to explain cases like the one shown in Fig. 6. Here the algorithm made a false positive error before the 3rd QRS and missed the following QRS. Formally, this is a false positive error, immediately followed by a false negative. However, if the time interval between these two detections is reasonably short, for example $\leq 200 \mathrm{~ms}$, we accepted this as one error only, labeled as Shifted False Positive Error (SP).

Another example of SP error as a result of artifacts just before the normal complexes is shown in Fig. 7.

SN - shifted negative error was assumed by the same principle as SP, but in the opposite sense. It also included twin $\mathrm{FN}+\mathrm{FP}$ error occurring within $200 \mathrm{~ms}$. The first incoming FP or FN error of the shifted is defining it as SP or SN.

The logic of using shifted errors (instead of FP and FN or FN and FP in cases when they appear within $200 \mathrm{~ms}$ of each other) is that thus the total number of beats in a record retains its value. Otherwise it would change depending on the type and number of errors and thus impede correct computation of Se and Sp.

The sensitivity Se is calculated by summing FN И SN, while the specificity $\mathrm{Sp}-$ by summing $\mathrm{FP}+\mathrm{SP}$.

$$
S e=\frac{T P}{T P+F N+S N}, \quad S p=\frac{T P}{T P+F P+S P} .
$$




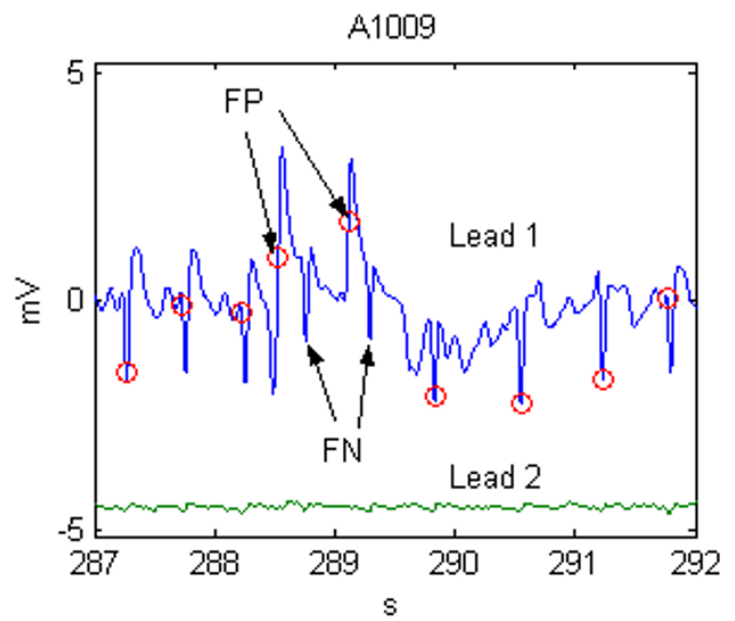

Figure 7

Shifted positive errors, false positive + false negative twins

The method was developed in Matlab. All 48 recordings from the MIT-BIH Arrhythmia database, without any exception, were used for testing the two algorithms.

The processed files containing detection marks were automatically compared with the original MIT-BIH annotated beats by specially designed software. It shows all cases where the annotation and detection marks differ by more than $60 \mathrm{~ms}$. These cases were examined by an independent expert, thus excluding possible author's influence.

The results for the two algorithms are presented in Table 1 .

Of all 110050 annotated beats ('unknown' or 'questionable' were excluded from the study), true detected are 109548 for Algorithm 1 and 109616 for Algorithm 2. The statistical indices are:

Algorithm 1: Se = $99.69 \%, \mathrm{Sp}=99.66 \%$;

Algorithm 2: $\mathrm{Se}=99.74 \%, \mathrm{Sp}=99.65 \%$.

The standard way of Se and Sp calculation,

$$
S e=\frac{T P}{T P+F N}, \quad S p=\frac{T P}{T P+F P},
$$

considering the joint SP and SN errors as two separate errors gives the following results:

Algorithm 1: Se = 99.54\%, Sp = 99.61\%;
Algorithm 2: Se = $99.60 \%, \mathrm{Sp}=99.60 \%$.

Algorithm 2 improved the sensitivity by $0.05 \%(0.06 \%$ for the standard evaluation) as a result of decreased number of undetected beats. This result can be observed for example in recordings 109,203, 210 and 223, where the additionally detected beats are respectively $6,9,12$ and 5 . The performance of both algorithms was especially tested with the file A5001 from the AHA containing Rover-T premature ventricular complexes, very close to the previous normal QRS complex (Fig. 5a). An improvement of 74 undetected by Algorithm 1 R-on-T complexes was observed. The detection of such premature ventricular complexes occurring at the time of ventricular repolarization was considered important, having in mind possible risk of ventricular fibrillation triggering by R-onT events.

\section{Conclusions}

The proposed algorithms for real-time and pseudo-realtime implementation are adaptive, independent of thresholds and constants values. They are self-synchronized to the QRS steep slope and the heart rhythm, regardless of the resolution and sampling frequency used. Due to the integration threshold, the algorithms are practically insensitive to electromyogram and similar high-frequency noise.

The algorithms can operate with one, two or more leads, using a combined lead signal derived from the sum of absolute values of the differentiated lead signals.

The statistical indices are higher than, or comparable to those, cited in the scientific literature. 
Table I: Statistical results for the two algorithms

\begin{tabular}{|c|c|c|c|c|c|c|c|c|c|c|c|}
\hline \multirow[t]{2}{*}{ File } & \multirow[t]{2}{*}{ Annotated beats } & \multicolumn{5}{|c|}{ Algorithm I } & \multicolumn{5}{|c|}{ Algorithm 2} \\
\hline & & $\mathrm{TP}$ & $\mathrm{FN}$ & FP & SN & $\mathrm{SP}$ & $\mathrm{TP}$ & $\mathrm{FN}$ & FP & SN & SP \\
\hline 100 & 2273 & 2273 & 0 & 0 & 0 & 0 & 2273 & 0 & 0 & 0 & 0 \\
\hline 101 & 1863 & 1862 & 1 & 4 & 0 & 0 & 1862 & I & 4 & 0 & 0 \\
\hline 102 & 2187 & 2187 & 0 & 0 & 0 & 0 & 2187 & 0 & 0 & 0 & 0 \\
\hline 103 & 2084 & 2062 & 2 & 54 & 11 & 9 & 2065 & 0 & 58 & 12 & 7 \\
\hline 104 & 2212 & 2211 & 1 & 0 & 0 & 0 & 2211 & 1 & 0 & 0 & 0 \\
\hline 105 & 2567 & 2543 & 2 & 35 & 8 & 14 & 2544 & 2 & 36 & 8 & 13 \\
\hline 106 & 2027 & 2017 & I & 1 & 0 & 9 & 2018 & 0 & 1 & 0 & 9 \\
\hline 107 & 2137 & 2135 & 2 & 0 & 0 & 0 & 2137 & 0 & 0 & 0 & 0 \\
\hline 108 & 1763 & 1664 & 2 & 40 & 3 & 94 & 1674 & I & 42 & 3 & 85 \\
\hline 109 & 2532 & 2521 & 11 & 1 & 0 & 0 & 2527 & 5 & 0 & 0 & 0 \\
\hline 111 & 2124 & 2124 & 0 & 0 & 0 & 0 & 2124 & 0 & 0 & 0 & 0 \\
\hline 112 & 2539 & 2539 & 0 & 0 & 0 & 0 & 2539 & 0 & 0 & 0 & 0 \\
\hline 113 & 1797 & 1797 & 0 & 0 & 0 & 0 & 1797 & 0 & 0 & 0 & 0 \\
\hline 114 & 1879 & 1879 & 0 & 0 & 0 & 0 & 1879 & 0 & 0 & 0 & 0 \\
\hline 115 & 1953 & 1951 & 0 & 4 & I & I & 1952 & 0 & 4 & 0 & 1 \\
\hline 116 & 2412 & 2389 & 22 & 2 & 0 & I & 2392 & 19 & 2 & 0 & 1 \\
\hline 117 & 1535 & 1535 & 0 & 0 & 0 & 0 & 1535 & 0 & 0 & 0 & 0 \\
\hline 118 & 2275 & 2275 & 0 & 0 & 0 & 0 & 2275 & 0 & 0 & 0 & 0 \\
\hline 119 & 1987 & 1987 & 0 & 0 & 0 & 0 & 1987 & 0 & 0 & 0 & 0 \\
\hline 121 & 1863 & 1863 & 0 & 0 & 0 & 0 & 1863 & 0 & 0 & 0 & 0 \\
\hline 122 & 2476 & 2476 & 0 & 0 & 0 & 0 & 2476 & 0 & 0 & 0 & 0 \\
\hline 123 & 1518 & 1516 & 2 & 0 & 0 & 0 & 1516 & 2 & 0 & 0 & 0 \\
\hline 124 & 1619 & 1617 & 2 & 0 & 0 & 0 & 1619 & 0 & 0 & 0 & 0 \\
\hline 200 & 2601 & 2549 & 9 & 39 & 18 & 25 & 2552 & 6 & 41 & 20 & 23 \\
\hline 201 & 1963 & 1902 & 60 & 0 & 0 & 1 & 1902 & 60 & 0 & 0 & I \\
\hline 202 & 2136 & 2130 & 6 & 0 & 0 & 0 & 2130 & 6 & 0 & 0 & 0 \\
\hline 203 & 2978 & 2901 & 71 & 13 & 3 & 3 & 2911 & 62 & 27 & 3 & 2 \\
\hline 205 & 2656 & 2652 & 4 & 0 & 0 & 0 & 2652 & 4 & 0 & 0 & 0 \\
\hline 207 & 1862 & 1860 & 2 & 0 & 0 & 0 & 1862 & 0 & I & 0 & 0 \\
\hline 208 & 2954 & 2937 & 14 & 7 & 2 & 1 & 2939 & II & 7 & 2 & 2 \\
\hline 209 & 3004 & 3004 & 0 & I & 0 & 0 & 3004 & 0 & I & 0 & 0 \\
\hline 210 & 2647 & 2591 & 56 & 1 & 0 & 0 & 2603 & 44 & 1 & 0 & 0 \\
\hline 212 & 2748 & 2748 & 0 & 0 & 0 & 0 & 2748 & 0 & 0 & 0 & 0 \\
\hline 213 & 3551 & 3548 & 3 & 0 & 0 & 0 & 3550 & 1 & 0 & 0 & 0 \\
\hline 214 & 2260 & 2258 & I & 1 & 1 & 0 & 2256 & 4 & I & 0 & 0 \\
\hline 215 & 3362 & 3362 & 0 & 0 & 0 & 0 & 3362 & 0 & 0 & 0 & 0 \\
\hline 217 & 2208 & 2204 & 3 & 0 & 0 & 1 & 2205 & 2 & 0 & 0 & 1 \\
\hline 219 & 2154 & 2153 & 1 & 0 & 0 & 0 & 2153 & 1 & 0 & 0 & 0 \\
\hline 220 & 2048 & 2048 & 0 & 0 & 0 & 0 & 2048 & 0 & 0 & 0 & 0 \\
\hline 221 & 2427 & 2426 & I & 0 & 0 & 0 & 2426 & 1 & 0 & 0 & 0 \\
\hline 222 & 2483 & 2480 & 2 & 0 & 0 & I & 2482 & 0 & 0 & 0 & I \\
\hline 223 & 2595 & 2585 & 10 & 0 & 0 & 0 & 2590 & 5 & 0 & 0 & 0 \\
\hline 228 & 2053 & 2053 & 0 & 0 & 0 & 0 & 2053 & 0 & I & 0 & 0 \\
\hline 230 & 2256 & 2256 & 0 & 0 & 0 & 0 & 2256 & 0 & 0 & 0 & 0 \\
\hline 231 & 1886 & 1886 & 0 & 0 & 0 & 0 & 1886 & 0 & 0 & 0 & 0 \\
\hline 232 & 1767 & 1766 & 0 & 12 & 0 & I & 1766 & 0 & 12 & 0 & 1 \\
\hline 233 & 3076 & 3073 & 3 & 0 & 0 & 0 & 3074 & 2 & 0 & 0 & 0 \\
\hline 234 & 2753 & 2753 & 0 & 0 & 0 & 0 & 2753 & 0 & 0 & 0 & 0 \\
\hline Sum & 110050 & 109548 & 294 & 215 & 47 & 161 & 109615 & 240 & 239 & 48 & 147 \\
\hline
\end{tabular}




\section{Acknowledgements}

The author gratefully acknowledges the contribution of Prof. Ivan Dotsinsky, Centre of Biomedical Engineering, Bulgarian Academy of Sciences, for examining and testing the results of the proposed algorithms.

\section{References}

I. Moreas JCTB, Seixas MO, Vilani FN, Costa EV: A QRS complex detection algorithm using electrocardiogram leads. Comp in Card 2002, 29:205-208.

2. Friesen GM, Jannett TC, Jadallah MA, Yates SL, Quint SR, Nagle HT: A comparison of the noise sensitivity of nine QRS detection algorithms. IEEE Trans on Biomed Eng 1990, 37:85-98.

3. Daskalov IK, Dotsinsky IA, Christov II: Developments in ECG acquisition, preprocessing, parameter measurement and recording. IEEE Eng in Med \& Biol 1998, 17:50-58.

4. Poli R, Cagnoni S, Valli G: Generic design of optimum linear and nonlinear QRS detection. IEEE Trans on Biomed Eng 1995, 42: $|137-||4|$.

5. Afonso VX, Tompkins WJ, Nguyen TQ, Luo S: ECG beat detection using filter banks. Trans Biomed Eng I999, 2:192-20I.

6. Dotsinsky I, Stoyanov T: Ventricular beat detection in single channel electrocardiograms. BioMed Eng OnLine 2004, 3:3 [http:/ /www.biomedical-engineering-online.com/content/3/1/3].

7. Englese WAH, Zeelenberg C: A single scan algorithm for QRS detection and feature extraction. IEEE Comp in Card 1979:37-42.

8. Pan J, Tompkins W: A real-time QRS detection algorithm. IEEE Trans Biomed Eng 1985, 32:230-236.

9. Ligtenberg A, Kunt M: A robust-digital QRS detection algorithm for arrhythmia monitoring. Computers and Biomed Res 1983, 16:273-286.

10. Li C, Zheng C, Tai C: Detection of ECG characteristic points using wavelet transforms. IEEE Trans on Biomed Eng 1995, 42:21-28.

II. Mark R, Moody G: MIT-BIH Arrhythmia data base directory. Cambridge: Massachusetts Institute of Technology 1988.

12. Levkov C: Orthogonal electrocardiogram derived from the limb and chest electrodes of the conventional 12-lead system. Med Biol Eng Comput 1987, 25: I55-164.

13. Bakardjian $\mathrm{H}$ : Ventricular beat classifier using fractal number clustering. Med Biol Eng Comput 1992, 30:495-502.

Publish with Bio Med Central and every scientist can read your work free of charge

"BioMed Central will be the most significant development for disseminating the results of biomedical research in our lifetime. "

Sir Paul Nurse, Cancer Research UK

Your research papers will be:

- available free of charge to the entire biomedical community

- peer reviewed and published immediately upon acceptance

- cited in PubMed and archived on PubMed Central

- yours - you keep the copyright

Submit your manuscript here:

http://www.biomedcentral.com/info/publishing_adv.asp
BiolMedcentral 